\title{
Genetic Characterization of Leaf and Stripe Rust Resistance in the Brazilian Wheat Cultivar Toropi
}

\author{
Silvia B. Rosa, ${ }^{1}$ Camila M. Zanella, ${ }^{2, \dagger}$ Colin W. Hiebert, ${ }^{3}$ Anita L. Brûlé-Babel, ${ }^{4}$ Harpinder S. Randhawa, ${ }^{5}$ \\ Stephen Shorter, ${ }^{6}$ Lesley A. Boyd, ${ }^{2}$ and Brent D. McCallum ${ }^{3, \dagger}$ \\ ${ }^{1}$ CÉROM, Centre de recherché sur les grains, 740 Chemin Trudeau, Saint-Mathieu-de-Beloeil, QC, J3G 0E2, Canada \\ 2 NIAB, Huntingdon Road, Cambridge, CB3 OLE, UK \\ ${ }^{3}$ Agriculture and Agri-Food Canada, Morden Research Centre, Morden, MB, R6M 1Y5, Canada \\ ${ }^{4}$ University of Manitoba, 66 Dafoe Road, Winnipeg, MB, R3T 2N2, Canada \\ ${ }^{5}$ Agriculture and Agri-Food Canada, Lethbridge Research Centre, 5403-1 Avenue South, Lethbridge, AB, T1J 4P4, Canada \\ ${ }^{6}$ Plant and Food Research, Canterbury Agriculture \& Science Centre, Gerald Street, Lincoln, New Zealand \\ Accepted for publication 25 June 2019.
}

\begin{abstract}
Leaf and stripe rust are major threats to wheat production worldwide. The effective, multiple rust resistances present in the Brazilian cultivar Toropi makes it an excellent choice for a genetic study of rust resistance. Testing of DNA from different seed lots of Toropi with 2,194 polymorphic 90K iSelect single nucleotide polymorphism markers identified significant genetic divergence, with as much as $35 \%$ dissimilarity between seed lots. As a result, further work was conducted with a single plant line derived from Toropi variant Toropi-6.4. A double haploid population with 168 lines derived from the cross Toropi- $6.4 \times$ Thatcher was phenotyped over multiple years and locations in Canada, New Zealand, and Kenya, with a total of seven field trials undertaken for leaf rust and nine for stripe rust. Genotyping

trait loci (QTL) derived from Toropi-6.4 were identified in multiple environments on chromosomes 1B (QLr.crc-1BL/QYr.crc-1BL), 3B (QLr.crc3BS), 4B (QYr.crc-4BL), 5A (QLr.crc-5AL and QYr.crc-5AL), and 5D (QLr.crc-5DS). The QTL QLr.crc-1BL/QYr.crc-1BL colocated with the multi-rust resistance locus $L r 46 / Y r 29$, while the QTL QLr.crc-5DS located to the $L r 78$ locus previously found in a wheat backcross population derived from Toropi. Comparisons of QTL combinations showed QLr.crc-1BL to contribute a significantly enhanced leaf rust resistance when combined with QLr.crc-5AL or QLr.crc-5DS, more so than when QLr.crc-5AL and QLr.crc$5 D S$ were combined. A strong additive effect was also seen when the stripe rust resistance QTL QYr.crc-1BL and QYr.crc-5AL were combined.
\end{abstract} with the $90 \mathrm{~K}$ iSelect array, simple sequence repeat and Kompetitive allelespecific polymerase chain reaction markers resulted in a genetic map of $3,043 \mathrm{cM}$, containing 1,208 nonredundant markers. Significant quantitative
Keywords: adult plant resistance, durable resistance, leaf rust, stripe rust, QTL, wheat
Wheat leaf (brown) and stripe (yellow) rust, caused by the fungal pathogens Puccinia triticina Eriks and P. striiformis Westend f. sp. tritici Eriks., respectively, are major global threats to wheat production (Pretorius et al. 2017). The introduction of rust resistance genes into elite wheat germplasm is the preferred method of disease control, being environmentally friendly and crucial to sustained wheat production. Many major, qualitative rust resistance genes have been identified in wheat. These are generally expressed through-out the life of the plant, being referred to as seedling or allstage resistance. However, these tend to represent race-specific resistance, and virulence shifts in the Puccinia population often result in the resistance genes becoming ineffective, frequently in a relatively short time frame after cultivar release. Quantitative rust resistance, identified as quantitative trait loci (QTL), tends to be

${ }^{\dagger}$ Corresponding authors: B. D. McCallum; Brent.McCallum@canada.ca, and C. M. Zanella; camila.zanella@niab.com

Funding: This study was supported by the Bilateral Biotechnology and Biological Sciences Research Council (BBSRC)-Embrapa Grant number BB/N01622X/1, the Agri-Food Research and Development Initiative, Natural Sciences and Engineering Research Council of Canada, and the Willy Wiebe Graduate Student Scholarship.

First and second authors contributed equally to this work.

*The $\boldsymbol{e}$-Xtra logo stands for "electronic extra" and indicates that five supplementary figures and five supplementary tables are published online.

The author(s) declare no conflict of interest.

This article is in the public domain and not copyrightable. It may be freely reprinted with customary crediting of the source. The American Phytopathological Society, 2019. partial and expressed postseedling, being referred to as adult plant resistance (APR) (Pinto-da-Silva et al. 2018; Rosewarne et al. 2013; Wang and Chen 2017). APR genes can be race-specific, but some have been demonstrated to be race-nonspecific, and are often associated with a slow-rusting phenotype (Caldwell 1968). Slowrusting resistance extends the latent period, reducing the size of uredinia (Singh et al. 1991, 2005). The APR loci Lr34/Yr18/ Sr57/Pm38, Lr46/Yr29/Sr58/Pm39, Lr67/Yr46/Sr55/Pm46, and Sr2/Yr30 are examples of partial, slow-rusting resistance with pleiotropic effect (Dyck 1987; Hare and McIntosh 1979; HerreraFoessel et al. 2011; Hiebert et al. 2010; Lillemo et al. 2008; McIntosh 1992; Rosewarne et al. 2006; Singh 1992; Singh et al. 2000; Spielmeyer et al. 2005), and in the case of $\operatorname{Lr} 34 / \mathrm{Yr} 18 / \mathrm{Sr} 57 /$ Pm38 and Lr67/Yr46/Sr55/Pm46 it is known that a single gene confers resistance to multiple pathogens (Krattinger et al. 2009; Moore et al. 2015). A better approach to sustainable rust resistance is therefore, the deployment of diverse resistance sources and multiple APR loci. A single QTL rarely confers adequate resistance, especially under high disease pressure, a combination of four or five QTL/genes being required to establish adequate levels of rust resistance (Singh et al. 2000).

The Brazilian cultivar Toropi, released in 1965, was commercially cultivated for over 15 years. Throughout its cultivation it maintained a high level of field resistance to leaf rust, although being seedling susceptible to all known Brazilian $P$. triticina isolates (Barcellos et al. 2000; Casassola et al. 2015; Zoldan and Barcellos 2002). Toropi originated from the cross Frontana 1971.37/Quaderna//Petiblanco 8. Frontana has been shown to contain the leaf rust APR genes $\operatorname{Lr} 13, \operatorname{Lr} 34$, and $\operatorname{Lr68}$ (HerreraFoessel et al. 2012; Singh and Rajaram 1992); however allelism 
tests have demonstrated that Toropi lacks Lr34 (Barcellos et al. 2000). Two leaf rust resistance genes were reported in Toropi on chromosomes 1AS and 4DS (Da-Silva et al. 2012). Recently, a leaf rust APR gene derived from Toropi was reported on chromosome 5DS (designated $L r 78$ ) (Kolmer et al. 2018), while three small effect QTL were found on chromosomes 1BL, 3BS, and 4BS in a separate population derived from Toropi (Kolmer et al. 2018). It has also been shown that Toropi has a prehaustorial resistance to leaf rust, with the majority of $P$. triticina infection sites being halted in their development before the formation of haustoria (Wesp-Guterres et al. 2013).

The leaf and stripe rust resistance in Toropi represents a valuable resource for wheat rust resistance breeding (Rosa et al. 2016). The durability of the resistance probably rests upon a combination of several genes. However, like many valuable wheat genotypes, seed of Toropi has been moved around the world, grown, and used by many breeders and researchers. Hence, the genetic purity of any Toropi seed lot is brought into question. The objectives of this study were therefore twofold. The first objective was to compare the genetic profiles of Toropi seed lots obtained from different sources to determine the extent Toropi genetics had drifted. Having selected a defined Toropi line, taken from a single plant showing good field leaf rust resistance, but seedling susceptibility, the second objective was to develop a doubled haploid mapping population and map field resistance to both leaf and stripe rust.

\section{MATERIALS AND METHODS}

Molecular genetic comparison between different seed lots of the Brazilian wheat cultivar Toropi. DNA of the Toropi seed lot Toropi-6.0 (originated from Brazilian Agricultural Research Corporation, EMBRAPA-Wheat, Brazil; Barcellos et al. 2000) and two lines derived from Toropi-6.0, Toropi-6.3, and Toropi-6.4, were provided by Agriculture and Agri-Food Canada (AAFC), Morden, Canada. Toropi-6.3 and Toropi-6.4 were selected from Toropi-6.0 following seedling inoculation with $P$. triticina race $\mathrm{BBBD}$, which indicated heterogeneity within the Toropi-6.0 seed lot. Toropi-6.4 and Toropi-6.3 were each selected from single plants, Toropi-6.4 being seedling susceptible to race BBBB, while Toropi-6.3 was resistant (Rosa et al. 2016). The SCAR markers SCS1302 and SCS1362 (Gupta et al. 2006) support the presence of Lr24 in Toropi-6.3, and absence from Toropi-6.4, Toropi-6.0, and Thatcher (data not show). AAFC also held seed of Toropi supplied by Silvia German, National Institute of Agricultural Research of Uruguay, Montevideo, Uruguay. This seed had been multiplied at AAFC and DNA was extracted from the Uruguay seed lots dated 2004, 2009, and 2011. DNA from Toropi was obtained from EMBRAPA-Wheat, Passo Fundo, Brazil (Toropi-EMBRAPA) and from Federal University of Rio Grande do Sul (UFRGS), Porto Alegre, Brazil (Toropi-UFRGS). Two, replicate DNAs of seed lots of Toropi were also provided by Evans Lagudah, Commonwealth Scientific and Industrial Research Organization (CSIRO), Australia.

In order to compare the genetic profiles of different sources of Toropi, DNA was isolated from 14-day-old single seed seedlings using the Qiagen DNeasy 96 Plant Kit (Qiagen, Mississauga, Ontario) and genotyped using the $90 \mathrm{~K}$ iSelect single nucleotide polymorphism (SNP) (Wang et al. 2014) array developed by Illumina Inc. (San Diego, CA). The SNP profiles of each wheat genotype were determined using GenomeStudio (Illumina). SNP markers that differed between two or more genotypes were retained for pairwise similarity analyses. Three biological replicates were genotyped of Toropi-6.0, Toropi-6.3, and Toropi-6.4. Two DNA samples were provided by CSIRO, but only one sample was used for Toropi-EMBRAPA, Toropi-UFRGS, and the 3 year samples from Uruguay. Thatcher was also included in the analysis as a genetic outlier. The pairwise similarity of the SNP profiles of each genotype were compared by an Euclidean distance analysis, the results being displayed in a heat-map plot using the package ggfortify (Tang et al. 2016) on R software (R Development Core Team, 2018).

Toropi mapping population development and genetic linkage map construction. Two crosses were made with the cultivar Thatcher: Toropi-6.4 $\times$ Thatcher and Toropi- $6.3 \times$ Thatcher. Double haploid (DH) populations were made from each cross using the maize/wheat hybridization method (Thomas et al. 2010), as summarized by Rosa et al. (2016). Three $F_{1}$ plants were used to generate each population. The Toropi- $6.4 \times$ Thatcher population had $171 \mathrm{DH}$ lines and the Toropi-6.3 $\times$ Thatcher was composed of $155 \mathrm{DH}$ lines.

The Toropi-6.4 $\times$ Thatcher DH population was genotyped using the 90K Illumina iSelect array (Wang et al. 2014), 227 polymorphic simple sequence repeat (SSR) (Somers et al. 2004) markers and 149 Kompetitive allele-specific polymerase chain reaction (KASP) (Allen et al. 2011) markers, genotyped by Kbioscience (LGC Genomics, Beverly, USA). For Illumina iSelect, DNA was extracted from a single plant from each DH line using the Qiagen DNeasy 96 Plant Kit (Qiagen, Mississauga, ON, Canada). SNP genotype calling were determined using GenomeStudio (Illumina). For SSR markers, DNA was extracted from multiple plants from each DH line using a modified ammonium acetate extraction (Chao and Somers 2012), and marker analysis carried out using Applied Biosystems ABI 3100 genetic analyzer (Applied Biosystems, Streetville, ON, Canada) as described by Somers et al. (2004). Data were converted to a gel-like image, using Genographer version 2.1.4, and GS500-LIZ or 500-ROX (Applied Biosystems, Foster City, California) as the internal molecular weight standards for the ABI 3100. The Toropi-6.4 $\times$ Thatcher DH population was also genotyped with the $\operatorname{cs} L V 46 G 22$ sequence tagged site (STS) marker, which is linked to Yr29/Lr46 (E. Lagudah, CSIRO Canberra, personal communication).

Redundant, cosegregating markers were removed using the BIN function of the IciMapping v. 4.1 software (Meng et al. 2015). Linkage analyses were conducted using MapDisto version 1.7.5.1 (Lorieux 2012) and the Kosambi (1943) mapping function with a minimal logarithm of the odds (LOD) threshold of 3.0 and $r=0.2$. Markers significantly distorted from the expected segregation ratio $(P<0.0001)$, as well as markers with more than $10 \%$ missing data, were excluded from the analysis. DH lines with more than $20 \%$ of missing data were also removed. The physical location of the SNP markers was confirmed using the Chinese Spring NRGene-IWGSC v1.0 genome assembly (https://www.wheatgenome.org/News/Latestnews/RefSeq-v1.0-URGI). MapChart v 2.3 (Voorrips 2002) was used to draw the linkage maps.

Field phenotypic evaluation of leaf and stripe rust resistance. Field trials of leaf and stripe rust resistance in the Toropi-6.4 $\times$ Thatcher DH population were undertaken in Canada, New Zealand, and Kenya. Leaf rust trials were conducted in Manitoba, Canada (Glenlea - 2010; Portage La Prairie - 2011 and 2012; and Morden - 2015, 2016, 2017, and 2018). Stripe rust trials were conducted in Canada (Creston, British Columbia - 2017 and 2018, and Lethbridge, Alberta - 2013, 2017 and 2018), in New Zealand (Lincoln - 2011 and 2012), and in Kenya (Njoro - 2012 and 2018). A randomized, complete block design, composed of two replicates was used in all field locations and seasons, the only exceptions being the field trials conducted at Creston in 2017 and Kenya in 2012, where there was only one replicate. The nurseries were organized in small rows of $60 \mathrm{~cm}$ in Canada, $1 \mathrm{~m}$ in New Zealand, and $70 \mathrm{~cm}$ in Kenya.

In Canada, the leaf rust spreader rows were composed of a mixture of the leaf rust susceptible wheat cultivars Morocco, Little Club, and Thatcher. Spreaders for stripe rust inoculation in Canada were composed of a mixture of susceptible cultivars Morocco, SWS18, AC Barrie, and Zak. In Canada, leaf rust spreader rows were inoculated by spraying urediospore mixtures of $P$. triticina isolates, suspended in light mineral oil (Bayol) on leaves at early tillering. The mixture of $P$. triticina races used represented the 
P. triticina population found in Canada in the previous year, in the proportion in which they were found in the Canadian P. triticina survey. There are typically about 40 races in this mixture. For stripe rust, the field trials conducted in Canada, New Zealand, and Kenya all relied on natural infection from the prevalent $P$. striiformis $\mathrm{f}$. sp. tritici populations.

Plants were scored for leaf and stripe rust infection at wheat anthesis, when the susceptible check lines showed over $50 \%$ rust infection. Leaf and stripe rust severity was scored according to the modified Cobb scale (Peterson et al. 1948; Roelfs et al. 1992), with infection type on the flag leaves being scored as $\mathrm{R}=$ resistant, $\mathrm{MR}=$ moderately resistant, $\mathrm{MS}=$ moderately susceptible, and $\mathrm{S}=$ susceptible (Knott 1989; McIntosh et al. 1995). In New Zealand and Kenya, leaf rust severity was recorded on a 0 to $100 \%$ severity infection scale.

The Toropi-6.3 $\times$ Thatcher DH population was phenotyped for leaf rust resistance in field trials in Canada (Glenlea - 2010, Portage La Prairie - 2011 and 2012) and for stripe rust in New Zealand (Lincoln - 2011 and 2012). The field experiments and phenotyping were conducted as described above for the Toropi- $6.4 \times$ Thatcher DH population.

Statistical analysis. An analysis of variance (ANOVA) was undertaken for the leaf and stripe rust percentage infection using the linear mixed model (REML) procedure in GenStat, 19th Edition (Rothamsted Experimental Station, Harpenden, UK). Predicted means for the Toropi- $6.4 \times$ Thatcher DH lines were extracted from the REML analyses for all phenotypic data set, with exception of Creston 2017 and Kenya 2012.

The mean percentage rust infections of each DH line, in each field trial, were displayed using the ggplot2 package in R (Wickham 2016). Pearson's correlation coefficients, across years and locations, were calculated for mean leaf and stripe rust percentage infection using psych (Revelle 2018), and plotted using corrplot (Wei and Simko 2017) packages in R. Significant differences

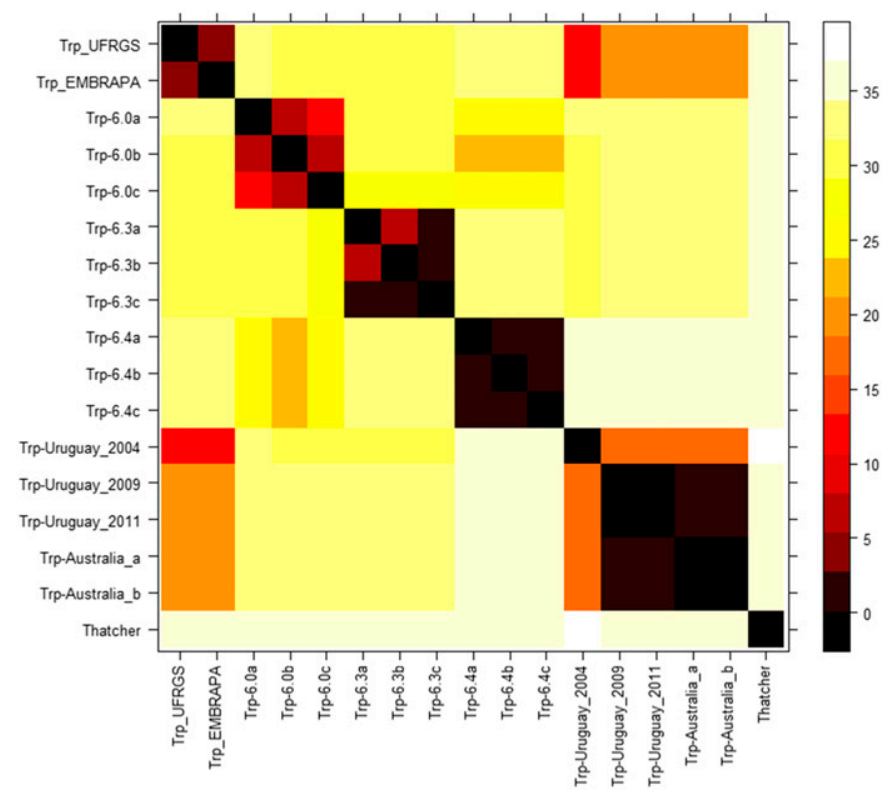

Fig. 1. A pairwise Euclidean analysis showing the genetic dissimilarities between 16 DNA samples of the wheat cultivar Toropi (Trp) and one DNA sample from Thatcher. The Toropi DNA is derived from different seed lots; Toropi-6.0, Toropi-6.3, and Toropi-6.4, AAFC, Morden, Canada; AAFC-ToropiUruguay, National Institute of Agricultural Research of Uruguay, Montevideo, Uruguay; Toropi-EMBRAPA, Embrapa-wheat, Passo Fundo, Brazil; Toropi-UFRGS, Federal University of Rio Grande do Sul, Porto Alegre, Brazil, and Toropi-Australia, CSIRO, Australia. Replicate DNA samples are indicated by lowercase letters. The color scale shows the percentage of 2,194 single nucleotide polymorphism markers that differ between each DNA sample. between the mean rust infection values of $\mathrm{DH}$ lines carrying the different QTL combinations were compared by ANOVA, followed by Tukey's HSD test (honestly significant difference, using a significance level of $P=0.05$ ), and were plotted using the agricolae package (Mendiburu 2017) in R.

Identification of leaf and stripe rust resistance loci in the cross Toropi-6.4 $\times$ Thatcher. QTL analysis was initially carried out using the mapping program IciMapping and further confirmed by R/qtl (Broman and Sen 2009). The inclusive composite interval mapping (ICIM) function was used to evaluate marker intervals associated with leaf and stripe rust resistance in the Toropi- $6.4 \times$ Thatcher DH population. Single marker regression (SMR), standard interval mapping (IM), Harley-Knott regression (HK), and composite interval mapping (CIM) were also performed using R/qtl. One thousand permutations for each data set, with a significance level of 0.05 , were performed to determine the LOD threshold. Stepwise regression was used to estimate both the main effects and percentages of phenotypic variance explained (PVE) of each significant QTL. For all environments, QTL analyses were conducted using both the arithmetic and predicted means. As we observed similar results with both the arithmetic and predicted means, only the QTL results using arithmetic mean are presented.

Conversion of SNP loci to KASP markers. SNP that were closely linked to the leaf or stripe rust resistance QTL on chromosomes 1B, 5A, and 5D were converted to KASP markers. The KASP markers were first evaluated for polymorphism between Toropi-6.4, Toropi-6.3 and Thatcher, and the polymorphic markers then mapped in the Toropi-6.4 $\times$ Thatcher and Toropi- $6.3 \times$ Thatcher populations.

KASP assays were performed as described by Kassa et al. (2016) and data analyzed using the KlusterCaller software (LGC Genomics). KASP markers were mapped in each population using SMR, $\mathrm{IM}$, and $\mathrm{HK}$ in $\mathrm{R} / \mathrm{qtl}$ for all leaf and stripe rust phenotypic data sets. One thousand permutations for each data set, with a significance level of 0.05 , were performed to determine the LOD threshold.

\section{RESULTS}

Comparison of the molecular genetic profiles of different seed sources of the Brazilian wheat cultivar Toropi. We tested DNA from different sources of Toropi seed using the $90 \mathrm{~K}$ iSelect SNP array to compare the molecular profile of each seed lot. A pairwise Euclidean analysis, using 2,194 polymorphic iSelect SNPs (all monomorphic SNP were not considered in the analysis), revealed genetic variation among the Toropi samples (Fig. 1; Supplementary Table S1). This analysis also confirmed the heterogeneity observed in Toropi-6.0 by Rosa et al. (2016) which led to the selection of the single plant lines Toropi-6.3 and Toropi-6.4. Toropi-6.3 differed from Toropi-6.0 by approximately $30 \%$, while Toropi-6.4 differed by $25 \%$. Toropi- 6.3 differed from Toropi- 6.4 by approximately $33 \%$. The sources of Toropi from Brazil (ToropiEMBRAPA and Toropi-UFRGS) had similar molecular profiles, differing from each other by approximately $4.4 \%$. This was expected, as both seed sources recently originated from EMBRAPA-Wheat. All three Toropi genotypes from AAFC, Morden differed from the genotypes from EMBRAPA and UFRGS by 30 to $34 \%$. The three sources of Toropi-Uruguay DNA were more closely related to the Brazilian seed sources (Toropi-EMBRAPA and Toropi-UFRGS; 10 to $20 \%$ dissimilarity) than to Toropi-6.0, 6.3, and 6.4 (30 to $35 \%$ dissimilarity). The DNA provided by CSIRO was also very similar in its molecular profile to the Uruguay 2009 and 2011 (1.4 to $1.7 \%$ dissimilarity) samples, but differed by $35 \%$ from Toropi- 6.4 used in this study to map leaf and stripe rust resistance.

Phenotypic evaluation of leaf and stripe rust resistance in the Toropi-6.4 $\times$ Thatcher population. The Toropi- $6.4 \times$ Thatcher DH population was phenotyped extensively for both leaf and stripe rust resistance in field trials conducted in multiple years 
in Canada, Kenya, and New Zealand. Seven field trials were conducted for leaf rust (Fig. 2A) and nine for stripe rust (Fig. 2B). In all seven leaf rust field trials, Toropi-6.4 expressed high levels of leaf rust resistance, with leaf rust infection ranging from 0 to $20 \%$. Some transgressive segregation was seen within the population, although mainly representing $\mathrm{DH}$ lines with greater leaf rust susceptibility compared with Thatcher, suggesting that Thatcher retains a low level of residual leaf rust resistance. Significant correlations were observed between all the leaf rust field trials (Supplementary Fig. S1; Supplementary Table S2).

Toropi-6.4 also exhibited high levels of stripe rust resistance, with levels of stripe rust infection ranging from 0 to $5 \%$ in the field trials carried out in Canada. However, in New Zealand (2011 to $40 \%$ and 2012 to $30 \%$ ) and Kenya (2012 to $20 \%$ and 2018 to $40 \%$ ) higher levels of stripe rust infection were seen on Toropi-6.4. In Canada and New Zealand, Thatcher was susceptible to stripe rust, with percentage infections ranging from 45 to $75 \%$ in Canada, and $55 \%$ (2012) and 90\% (2018) in New Zealand. However, in Kenya, Thatcher showed moderate resistance to stripe rust (2012 to $20 \%$ and 2018 to $30 \%$ ). Transgressive segregation for stripe rust resistance was observed in all field trials, although DH lines more resistant than Toropi-6.4 were more prominent in Kenya and New Zealand, where Toropi-6.4 did not confer complete stripe rust resistance, and Thatcher exhibited moderate resistance. Significant correlations $(P<0.0001)$ were observed between all the stripe rust field trials undertaken in Canada. The stripe rust trials undertaken in New Zealand in 2011 and 2012, and in Kenya in 2012 and 2018, also correlated in-country. However, the 2018 Kenya trial did not correlate at $P<0.0001$ with the stripe rust trial undertaken in Creston in 2017 and in New Zealand in 2012 (Supplementary Fig. S1B and Table S2).

Construction of a genetic linkage map for the Toropi$6.4 \times$ Thatcher population. Three Toropi- $6.4 \times$ Thatcher DH lines were removed due to low quality or high percentage of missing data, leaving $168 \mathrm{DH}$ lines for genetic linkage map construction. From the $90 \mathrm{~K}$ SNP array 10,576 SNPs were retained after quality control and removal of markers with more than $10 \%$ missing data. After removal of markers showing segregation distortion we were left with 9,808 markers. The BIN function of IciMapping was used to remove cosegregating markers, leaving 1,301 markers from which the genetic linkage map was constructed. The final linkage map was composed of 1,208 marker loci (1,101 SNP, 22 KASP, and 85 SSR markers), forming 21 linkage groups, representing all 21 wheat chromosomes (Supplementary Fig. S2). The linkage map had a total length of 3,043 cM, with an average interval between marker loci of $2.51 \mathrm{cM}$. The A genome had the most markers $(45.36 \%)$, followed by the B (40.89\%) and the D genome (13.74\%) (Supplementary Table S3).

QTL analysis of leaf and stripe rust resistance in the Toropi-6.4 $\times$ Thatcher cross. Six QTL conferring leaf rust resistance and four QTL conferring stripe rust resistance were identified in the Toropi-6.4 $\times$ Thatcher DH population (Fig. 3; Table 1). QTL contributing to leaf rust resistance in Toropi-6.4 were found on chromosomes 1BL, 3BS, 5AL and 5DS. The QTL located on chromosomes $1 \mathrm{BL}$ and $5 \mathrm{AL}$ showed strong leaf rust resistance effects. QLr.crc-1BL was detected in all seven field experiments, with the highest LOD value (LOD 19.10) being seen in the 2016 trial in Morden, Canada. QLr.crc-5AL was detected in three field trials in Canada (Glenlea 2010, Morden 2015, and 2018), with the highest LOD value (LOD 16.35) being seen at Glenlea 2010.

The smaller effect QTL QLr.crc-5DS (max. LOD 6.98) was detected in five field trials in Canada (Portage 2012, Morden 2015, 2016, 2017, and 2018). The leaf rust resistance QTL on chromosome 3B (QLr.crc-3BS) was detected in three field trials in Canada (Portage 2012, Morden 2016 and 2018), having a maximum LOD value of 6.17 (Morden 2015).

Surprisingly, two minor leaf rust resistance QTL were detected in the Toropi-6.4 $\times$ Thatcher population derived from Thatcher: QLr.crc-2BL and QLr.crc-3DS. QLr.crc-2BL was identified in Portage la Prairie in 2012 (LOD = 3.96) and Morden in $2018(\mathrm{LOD}=4.29)$. QLr.crc-3DS was identified in Portage la Prairie in 2012 (LOD = 4.41) and Morden in $2016(\mathrm{LOD}=3.04)$ and $2018(\mathrm{LOD}=5.22)$.

Three of the four QTL conferring stripe rust resistance in the Toropi-6.4 $\times$ Thatcher population were derived from Toropi-6.4: QYr.crc-1BL, QYr.crc-4BL, and QYr.crc-5AL (Fig. 2). The QTL found on chromosomes $1 \mathrm{BL}$ and $5 \mathrm{AL}$ showed strong effects, QYr.crc-1BL being found in four field trials (Lethbridge 2013, New Zealand 2011 and 2012, and Kenya 2018), with the highest LOD values being seen in the New Zealand 2012 field trial data set (LOD = 12.66). QYr.crc-1BL colocated with the leaf rust resistance QTL
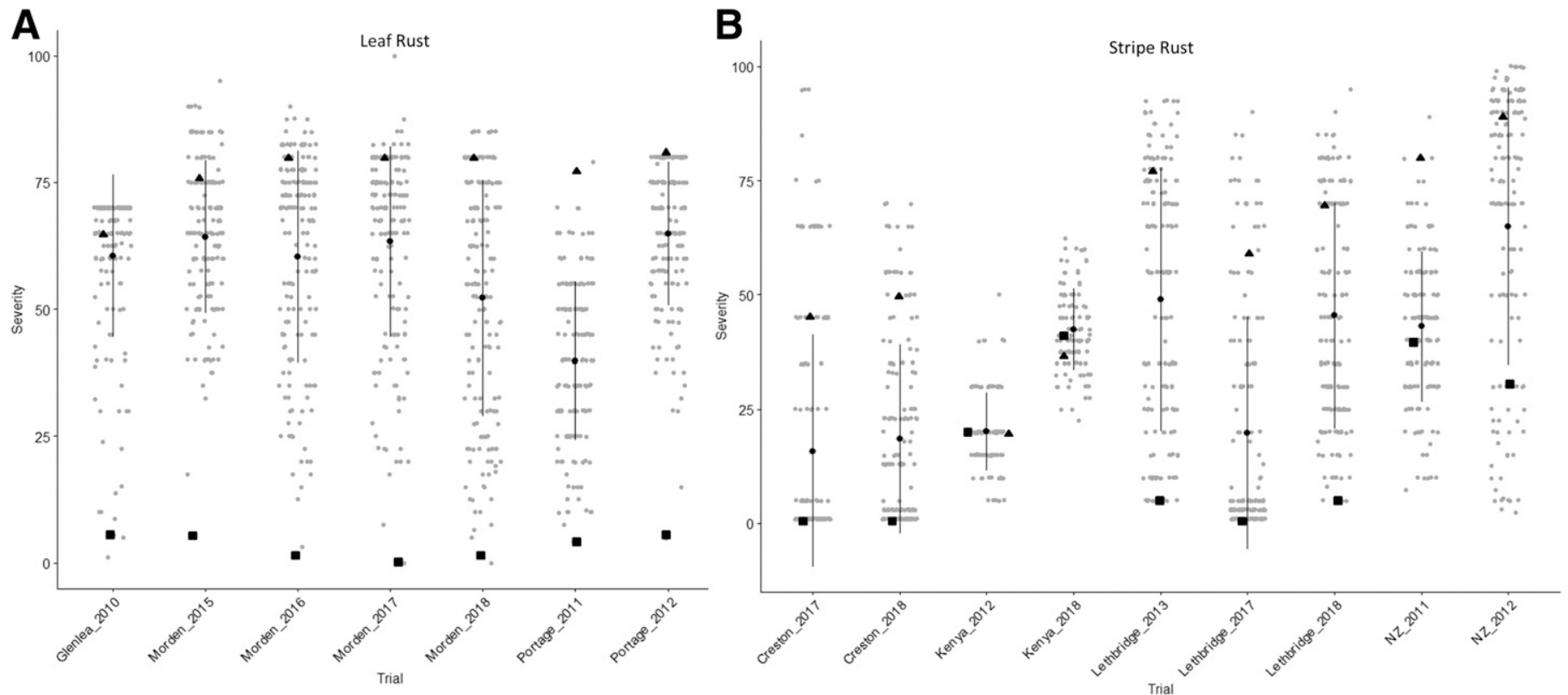

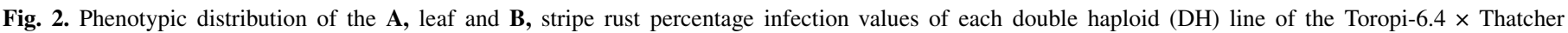

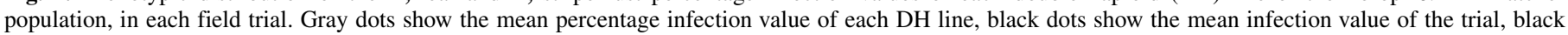

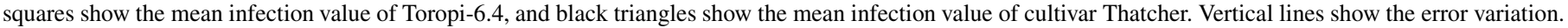
New Zealand (NZ). 
QLr.crc- $1 B L$, cosegregating with STS marker csLV46. QYr.crc-5AL was detected in eight of the nine stripe rust field trials (Lethbridge 2013, 2017, and 2018; Creston 2017 and 2018; New Zealand 2011 and 2012; and Kenya 2012). The highest LOD value was found in Lethbridge 2013 (LOD =42.84). QYr.crc-4BL was only found in the Lethbridge 2018 trial $(\mathrm{LOD}=7.44)$. QYr.crc-2BS, derived from Thatcher, was only detected in the Kenyan 2018 stripe rust field trial $(\mathrm{LOD}=11.03)$.

Validation of leaf and stripe rust resistance QTL in Toropi-6.3. The $90 \mathrm{k}$ SNP array markers linked with the significant leaf and stripe rust QTL, QLr.crc-1BL/QYr.crc-1BL, QYr.crc.5AL, QLr.crc.5AL, and QLr.crc.5DS were converted to KASP markers. A total of 18 SNP markers were selected, of which 14 amplified and were polymorphic between the parents in both the Toropi- $6.4 \times$ Thatcher and Toropi-6.3 $\times$ Thatcher DH populations (Supplementary Table S4). The KASP markers were screened in both populations.

The Toropi- $6.3 \times$ Thatcher population was phenotyped for both leaf and stripe rust resistance in Canada and New Zealand. In all three leaf rust field trials Toropi-6.3 expressed high levels of leaf rust resistance, and some transgressive segregation was seen between the DH lines. With stripe rust, Toropi-6.3 exhibited moderated levels of resistance in the New Zealand field trials (2011 to $35 \%$ and 2012 to $40 \%$ ) (Supplementary Fig. S3).

Single marker regressions were performed to (i) confirm comapping of the KASP markers with the respective QTL in the Toropi-6.4 $\times$ Thatcher population and (ii) determine whether the QTL QLr.crc-1BL/QYr.crc-1BL, QYr.crc.5AL, QLr.crc.5AL, and QLr.crc.5DS were present in Toropi-6.3. The KASP markers defining each QTL produced significant associations $(P<0.05)$ when mapped in the Toropi-6.4 $\times$ Thatcher population, confirming the presence of each QTL. In the Toropi-6.3 $\times$ Thatcher population the KASP markers detected QLr.crc-1BL/QYr.crc-1BL and QLr.crc$5 A L$, but not QLr.crc-5DS and QYr.crc-5AL (Supplementary Table S5).
Interactions between QTL. The effect of QTL interactions on leaf and stripe rust resistance was evaluated. The average leaf rust percentage infection of lines containing different combinations of the leaf rust resistance QTL QLr.crc-1BL, QLr.crc-5AL, and QLr.crc-5DS were compared (Supplementary Fig. S4). Alone, each QTL had only a small effect on the level of leaf rust infection. However, a clear additive effect was seen when these QTL were combined, with lines containing all three leaf rust QTL having the least leaf rust infection. While variation existed across field trials, the general trend was for higher levels of leaf rust resistance when QLr.crc-1BL was combined with QLr.crc-5AL or QLr.crc-5DS than when $Q L r . c r c-5 A L$ and $Q L r . c r c-5 D S$ were combined, as exemplified in Figure 4A.

A strong additive effect was seen in most field trials when the stripe rust resistance QTL QYr.crc-1BL and QYr.crc-5AL were combined (except Kenya 2018) (Supplementary Fig. S5). Alone, each QTL significantly reduced stripe rust infection levels in Lethbridge in 2013 and in 2017 and Kenya in 2012, but only QYr.crc-5AL had a significant effect in most field trails, while in Kenya 2018 neither QTL significantly reduced stripe rust infection levels. In Canada, QYr.crc-5AL had a significant effect in all field trials, while QYr.crc-1BL was only significant at Lethbridge 2013 and 2017, as exemplified in Figure 4B.

\section{DISCUSSION}

The field leaf rust resistance in the old Brazilian wheat cultivar Toropi is considered by many to be different from other sources of durable resistance. Released in 1965, Toropi is known to be susceptible at the seedling stage to all known Brazilian isolates of P. triticina (Marcia Chaves, personal communication). In the field Toropi still confers very good levels of leaf rust resistance, having been tested extensively in Brazil, Canada, and the United States (Barcellos et al. 2000, Rosa et al. 2016; Kolmer et al. 2018). However, efforts to identify the location of the genes/QTL
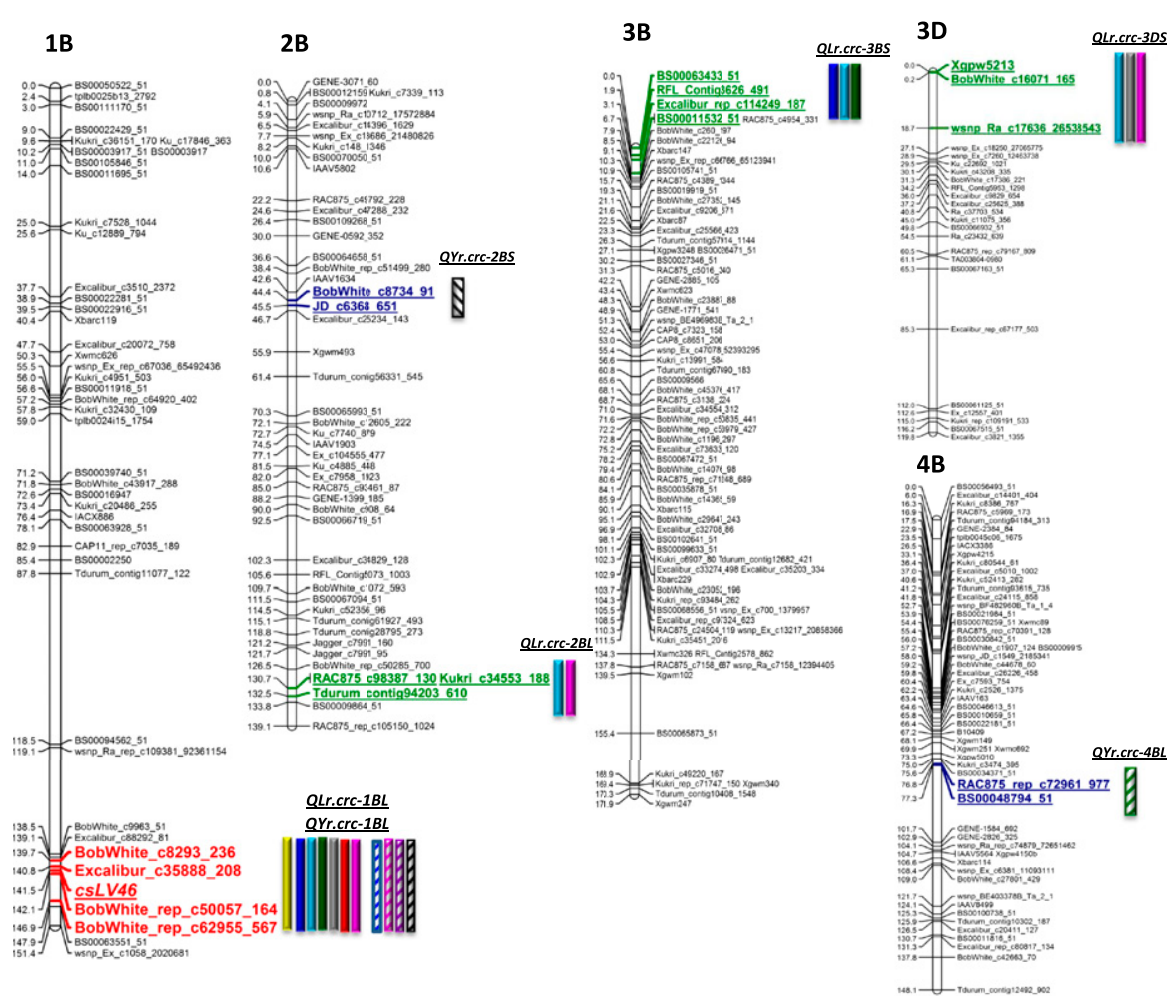

$5 A$

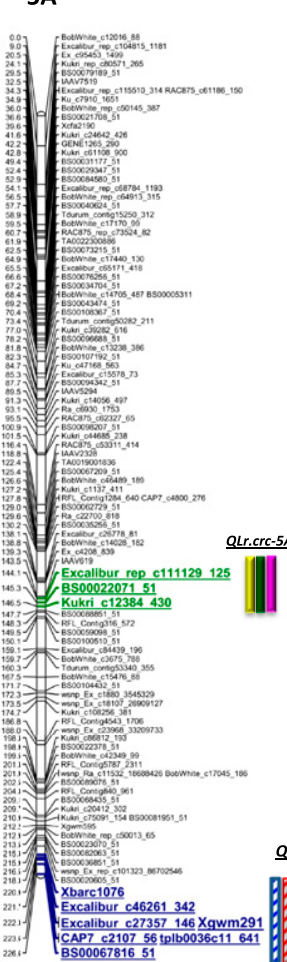

5D

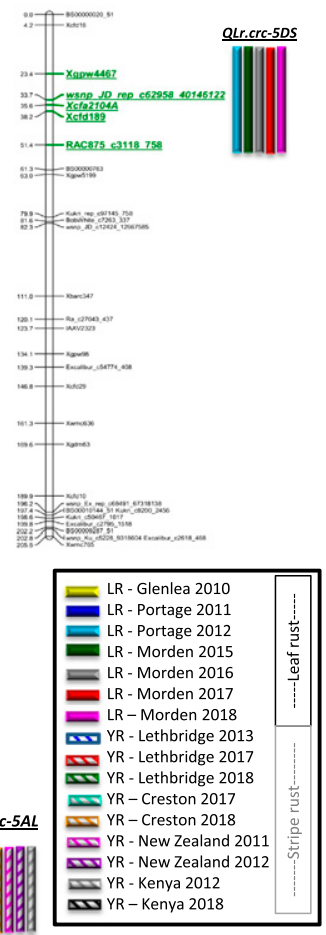

Fig. 3. Leaf and stripe rust resistance quantitative trait loci (QTL) identified in the Toropi- $6.4 \times$ Thatcher population. Six and four significant QTL for leaf (green) and stripe (blue) rust resistance, respectively, as identified across seven leaf rust and nine stripe rust field trials, are shown against their chromosome locations on the genetic linkage map of Toropi-6.4 × Thatcher. The markers significantly linked with QTL are highlighted in green for leaf rust, in blue for stripe rust, and in red for both. 
conferring the leaf rust resistance in Toropi have been difficult, with the reported results showing inconsistencies.

Two leaf rust resistance genes were originally reported by DaSilva et al. (2012) on chromosomes 1AS and 4DS based on monosomic and single marker regression analyses. Kolmer et al. (2018) reported a leaf rust APR gene on chromosome 5DS, designated $L r 78$, in one backcross population, and another three, small effect QTL on chromosomes 1BL ( $L r 46 / Y r 29)$, 3BS and 4BS in a second population derived from Toropi. In addition, good levels of stripe rust resistance have subsequently been found in Toropi (Rosa et al. 2016), but to the best of our knowledge no studies have yet been reported on the genetics underlying this stripe rust resistance.

Consequently, seed of Toropi has changed hands many times, having been worked on by many groups around the world. As a result, the genetic purity of Toropi has been bought into question. The Toropi seed lots obtained from Brazil were genetically very different from seed lots that had been multiplied over many years in other institutes around the world. This would indicate that significant levels of residual genetic diversity can exist within any given seed lot of a cultivar of wheat, an inbred, homozygous species, and how easy it is for wheat cultivars to genetically diverge during standard seed multiplication procedures. The genetic variation found between different seed lots of Toropi would also go some way to explaining the inconsistencies reported in the literature regarding the leaf rust resistance genes/QTL present in Toropi.
Extensive phenotyping for leaf rust, over many years and locations, indicated that Toropi-6.4 provided effective resistance to the $P$. triticina populations prevalent across Canada. Four QTL for leaf rust were identified in Toropi-6.4, QLr.crc-1BL, QLr.crc$3 B S, Q L r . c r c-5 A L$, and QLr.crc-5DS. While variation was seen across years and locations, $Q L$ r.crc- $1 B L$ was detected in all field leaf rust data sets. $Q L$ L.crc- $1 B L$ cosegregated with the marker $c s L V 46$, which has previously been shown to be linked to the rust resistance locus Lr46/Yr29 (E. S. Lagudah, personal communication). As seedling tests with 12 different Canadian $P$. triticina races had all resulted in susceptible phenotypes the field resistance seen in Toropi-6.4 is likely to be race-nonspecific (Rosa et al. 2016).

Lr46 is unique in that it confers broad-spectrum resistance to four biotrophic fungal pathogens: P. triticina (Lr46), P. striiformis f. sp. tritici (Yr29), P. graminis f. sp. tritici (Sr58) (Singh et al. 2013), and Blumeria graminis f. sp. tritici (Pm39) (Lillemo et al. 2008). Lr46 has been reported in 20 studies from 19 wheat donor genotypes (Pinto da Silva et al. 2018), with Lr46 being associated with the marker csLV46 in 13 of these studies. The effectiveness of $L r 46$ depends greatly upon the genetic background and environment (Lan et al. 2015). $\mathrm{Lr} 46$ explained from 2.9 to $74.5 \%$ of the total PVE in the 20 reported studies (Pinto da Silva et al. 2018). In this study $Q L r$. crc- $1 B L$ explained between 1.76 and $16.61 \%$ of the PVE. Alone, $Q L$ r.crc-1BL did not significantly reduce leaf rust infection levels, but in combination with either QLr.crc-5AL or QLr.crc-5DS made a significant contribution to resistance. Similarly, $L r 46$ was shown to

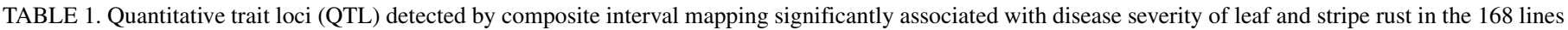
of the Toropi- $6.4 \times$ Thatcher doubled haploid population evaluated from 2010 to 2018 in a total of seven trials for leaf rust, and nine trials for stripe rust ${ }^{\mathrm{a}}$

\begin{tabular}{|c|c|c|c|c|c|c|c|c|c|}
\hline QTL & Location & Chromosome & Position (cM) & Left marker & Right marker & $\mathrm{LOD}^{\mathrm{b}}$ & $\begin{array}{c}\% \\
\mathrm{PVE}^{\mathrm{c}}\end{array}$ & Add $^{\mathrm{d}}$ & Source \\
\hline \multicolumn{10}{|l|}{ Leaf rust } \\
\hline \multirow{7}{*}{ QLr.crc- $1 B L$} & Glenlea_2010 & $1 \mathrm{BL}$ & \multirow[t]{7}{*}{$140.8-146.1$} & Excalibur_c35888_208 & $\operatorname{csLV46}$ & $3.79(4.69)$ & 1.76 & 4.07 & Trp-6.4 \\
\hline & Portage_2011 & $1 \mathrm{BL}$ & & $\operatorname{csLV} 46$ & BobWhite_rep_c50057_164 & $4.89(4.45)$ & 13.74 & 9.56 & Trp-6.4 \\
\hline & Portage_2012 & $1 \mathrm{BL}$ & & csLV46 & BobWhite_rep_c62955_567 & $5.09(5.85)$ & 16.61 & 9.93 & Trp-6.4 \\
\hline & Morden_2015 & $1 \mathrm{BL}$ & & $\operatorname{csLV} 46$ & BobWhite_rep_c62955_567 & $14.07(12.1)$ & 10.53 & 7.22 & Trp-6.4 \\
\hline & Morden_2016 & $1 \mathrm{BL}$ & & $\operatorname{csLV} 46$ & BobWhite_rep_c62955_567 & $19.10(18.8)$ & 13.10 & 13.91 & Trp-6.4 \\
\hline & Morden_2017 & $1 \mathrm{BL}$ & & $\operatorname{csLV} 46$ & BobWhite_rep_c62955_567 & $13.01(13.3)$ & 10.76 & 9.51 & Trp-6.4 \\
\hline & Morden_2018 & $1 \mathrm{BL}$ & & $\operatorname{csLV} 46$ & BobWhite_rep_c62955_567 & $14.23(14.3)$ & 3.22 & 15.56 & Trp-6.4 \\
\hline \multirow[t]{2}{*}{ QLr.crc- $2 B L$} & Portage_2012 & $2 B L$ & \multirow[t]{2}{*}{$130.6-132.5$} & Kukri_c34553_188 & Tdurum_contig94203_610 & $3.96\left(2.54^{*}\right)$ & 11.12 & -8.08 & Thatcher \\
\hline & Morden_2018 & $2 \mathrm{BL}$ & & Kukri_c34553_188 & Tdurum_contig94203_610 & $4.29\left(4.17^{*}\right)$ & 2.78 & -13.25 & Thatcher \\
\hline \multirow[t]{3}{*}{ QLr.crc-3BS } & Portage_2011 & 3BS & \multirow[t]{3}{*}{$0.0-6.64$} & BS00063433_51 & RFL_Contig3626_491 & $2.54(2.74 *)$ & 1.86 & 3.53 & Trp-6.4 \\
\hline & Portage_2012 & 3BS & & Excalibur_rep_c114249_187 & BS00011532_51 & $3.61(2.37 *)$ & 2.32 & 3.31 & Trp-6.4 \\
\hline & Morden_2015 & 3BS & & Excalibur_rep_c114249_187 & BS00011532_51 & $6.17(6.07)$ & 3.96 & 4.10 & Trp-6.4 \\
\hline \multirow[t]{3}{*}{ QLr.crc-3DS } & Portage_2012 & 3DS & \multirow[t]{3}{*}{$0.00-18.7$} & BobWhite_c16071_165 & wsnp_Ra_c17636_26538543 & $4.41(3.01 *)$ & 2.87 & -3.64 & Thatcher \\
\hline & Morden_2016 & 3DS & & BobWhite_c16071_165 & wsnp_Ra_c17636_26538543 & $3.04(2.91 *)$ & 1.27 & -4.53 & Thatcher \\
\hline & Morden_2018 & 3DS & & BobWhite_c16071_165 & wsnp_Ra_c17636_26538543 & $5.22(5.95)$ & 0.66 & -6.78 & Thatcher \\
\hline \multirow[t]{3}{*}{ QLr.crc-5AL } & Glenlea_2010 & $5 \mathrm{AL}$ & \multirow[t]{3}{*}{$144.1-146.52$} & Excalibur_rep_c111129_125 & Kukri_c12384_430 & $16.35(14.3)$ & 16.78 & 16.52 & Trp-6.4 \\
\hline & Morden_2015 & $5 \mathrm{AL}$ & & Excalibur_rep_c111129_125 & Kukri_c12384_430 & $4.53(4.40)$ & 2.89 & 3.67 & Trp-6.4 \\
\hline & Morden_2018 & $5 \mathrm{AL}$ & & Excalibur_rep_c111129_125 & Kukri_c12384_430 & $2.69 *(1.97 *)$ & 2.73 & 13.12 & Trp-6.4 \\
\hline \multirow[t]{5}{*}{ QLr.crc-5DS } & Portage_2012 & 5DS & \multirow[t]{5}{*}{$23.35-51.4$} & Xcfa2104-5D & $X c f d 189-5 D$ & $6.42(3.17 *)$ & 4.63 & 4.62 & Trp-6.4 \\
\hline & Morden_2015 & 5DS & & Xcfd189-5D & RAC875_c3118_758 & $4.65\left(3.75^{*}\right)$ & 3.24 & 3.66 & Trp-6.4 \\
\hline & Morden_2016 & 5DS & & Xgpw4467.5DS & wsnp_JD_rep_c62958_40146122 & $3.34(4.36)$ & 1.41 & 4.77 & Trp-6.4 \\
\hline & Morden_2017 & 5DS & & Xgpw4467.5DS & wsnp_JD_rep_c62958_40146122 & $2.98 *\left(3.56^{*}\right)$ & 1.40 & 4.49 & Trp-6.4 \\
\hline & Morden_2018 & 5DS & & Xgpw $4467.5 D S$ & wsnp_JD_rep_c62958_40146122 & $6.98(6.99)$ & 1.07 & 8.18 & Trp-6.4 \\
\hline \multicolumn{10}{|l|}{ Stripe rust } \\
\hline \multirow[t]{4}{*}{ QYr.crc- $1 B L$} & Lethbridge_2013 & $1 \mathrm{BL}$ & \multirow[t]{4}{*}{$139.65-146.1$} & BobWhite_c8293_236 & $\operatorname{csLV} 46$ & $5.09(5.70)$ & 4.91 & 6.74 & Trp-6.4 \\
\hline & New Zealand_2011 & $1 \mathrm{BL}$ & & $\operatorname{csLV46}$ & BobWhite_rep_c62955_567 & $7.15(6.83)$ & 8.41 & 0.54 & Trp-6.4 \\
\hline & New Zealand_2012 & $1 \mathrm{BL}$ & & BobWhite_c8293_236 & $\operatorname{csLV} 46$ & $18.26(21.1)$ & 12.66 & 22.58 & Trp-6.4 \\
\hline & Kenya_2018 & $1 \mathrm{BL}$ & & $\operatorname{csLV} 46$ & BobWhite_rep_c62955_567 & $5.61(4.64)$ & 8.09 & 3.29 & Trp-6.4 \\
\hline QYr.crc- $2 B S$ & Kenya_2018 & 2BS & $44.34-45.53$ & BobWhite_c8734_91 & JD_c6368_651 & $11.03(8.12)$ & 17.78 & -4.87 & Thatcher \\
\hline QYr.crc $-4 B L$ & Lethbridge_2018 & $4 \mathrm{BL}$ & $76.7-77.3$ & RAC875_rep_c72961_977 & BS00048794_51 & $7.44(7.79)$ & 19.97 & 11.79 & Trp-6.4 \\
\hline \multirow[t]{8}{*}{ QYr.crc-5AL } & Lethbridge_2013 & $5 \mathrm{AL}$ & \multirow[t]{8}{*}{$220.8-225.7$} & tplb0036c11_641 & BS00067816_51 & $42.84(45.3)$ & 56.05 & 22.69 & Trp-6.4 \\
\hline & Lethbridge_2017 & $5 \mathrm{AL}$ & & CAP7_c2107_56 & tplb0036c11_641 & $16.92(15.9)$ & 6.76 & 24.36 & Trp-6.4 \\
\hline & Lethbridge_2018 & $5 \mathrm{AL}$ & & tplb0036c11_641 & BS00067816_51 & $18.84(14.1)$ & 29.81 & 14.39 & Trp-6.4 \\
\hline & Creston_2017 & $5 \mathrm{AL}$ & & Xgwm291 & CAP7_c2107_56 & $10.20(10.9)$ & 24.59 & 12.57 & Trp-6.4 \\
\hline & Creston_2018 & $5 \mathrm{AL}$ & & tplb0036c11_641 & BS00067816_51 & $14.57(15.9)$ & 17.17 & 11.10 & Trp-6.4 \\
\hline & New Zealand_2011 & $5 \mathrm{AL}$ & & Excalibur_c27357_146 & Xgwm291 & $15.48(13.2)$ & 20.63 & 0.85 & Trp-6.4 \\
\hline & New Zealand_2012 & $5 \mathrm{AL}$ & & tplb0036c11_641 & BS00067816_51 & $12.90(14.2)$ & 5.30 & 14.34 & Trp-6.4 \\
\hline & Kenya_2012 & $5 \mathrm{AL}$ & & Excalibur_c27357_146 & CAP7_c2107_56 & $7.58(7.85)$ & 9.51 & 4.53 & Trp-6.4 \\
\hline
\end{tabular}

a Logarithm of the odds scores (LOD) were showing IciMapping and R/qtl (between brackets) results.

b Nonsignificant LOD score at level at $P=0.05$.

c PVE, percentage of phenotypic variance explained by individual QTL.

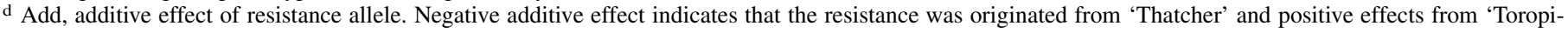
$6.4^{\prime}$. 
enhance leaf rust resistance when combined with the QTL QLr.cdl$5 B L$ derived from the wheat cultivar Americano 25e (Kolmer 2015).

QLr.crc-5AL was detected in three of the seven field trials and QLr.crc-5DS in five field trials. QLr.crc-5AL represents a new leaf rust QTL, and to the best of our knowledge no leaf rust resistant locus has been reported in this region of the long arm of chromosome 5A (Pinto da Silva et al. 2018). A minor QTL, QLr. cimmyt-5AL, derived from cultivar Avocet has been reported on 5AL (Rosewarne et al. 2012), but it does not colocate with QLr.crc-5AL.

QLr.crc-5DS lies in the same region on chromosome 5DS where Kolmer et al. (2018) found the leaf rust resistance gene $L r 78$. The 9K SNP marker IWA6289, which defines $L r 78$ (Kolmer et al. 2018), is the same SNP as the 90K SNP marker wsnp_JD_rep_ c62958_40146122 that defines QLr.crc-5DS. While neither $Q L r$. crc-5AS nor $Q L r . c r c-5 D S$ alone had a significant effect on leaf rust infection levels, their value to wheat rust resistance breeding is seen when combined with other QTL, such as QLr.crc-1BL.

QLr.crc-3BS was detected in three field trials. Kolmer et al. (2018) also found a leaf rust resistance QTL derived from Toropi on the short arm of chromosome 3B (QLr.cdl-3BS.2), the closest marker locus to the QTL peak being Xbarc147. Xbarc147 was mapped in the Toropi- $6.4 \times$ Thatcher population, laying $6.0 \mathrm{cM}$ from the peak marker defining QLr.crc-3BS. Li et al. (2017) also identified a QTL on 3BS (QLr.hwwg-3BS.1) in cultivar Clark, which was provisionally assigned as $L r 74$. $L r 74$ was closely linked to marker loci Xgwm533 and Xbarc147, placing QLr.crc-3BS and $L r 74$ within a $20 \mathrm{cM}$ interval.

Lower levels of stripe rust infection were seen on Toropi-6.4 in Canada compared with New Zealand and Kenya. As infection in the stripe rust field trials relied on natural $P$. striiformis $\mathrm{f}$. sp. tritici inoculum, these differences in infection levels may be due to differences in $P$. striiformis f. sp. tritici inoculum levels. Also the environmental conditions for stripe rust infection development may have differed between Canada, New Zealand, and Kenya, New Zealand and Kenya generally being considered stripe rust areas. However, it may also indicate a race-specific response conferred by one or more of the stripe rust resistance genes in Toropi-6.4. Three QTL for stripe rust resistance, QYr.crc-1BL, QYr.crc- $4 B L$, and QYr.crc-5AL were identified in Toropi-6.4. As with QLr.crc-1BL, QYr.crc-1BL located to the region on the long arm of chromosome 1 A where the multirust resistance locus $L r 46 / Y r 29$ is located. QYr.crc- $1 B L$ was only detected in four of the nine field trials, being most effective in New Zealand (2011 and 2012) and Kenya 2018. In Canada QYr.crc-1BL was only detected in the Lethbridge 2013 field trial. However, when combined with $Q Y$ r.crc-5AL a clear additive effect was observed.

QYr.crc-5AL was detected in eight of the nine stripe rust field trials. QYr.crc-5AL maps to the same region on the long arm of chromosome 5A as the Yr48/Yr34 locus (Lowe et al. 2011; Qureshi et al. 2018). The APR Yr48 was identified in the synthetic spring wheat PI610750 (pedigree Croc1/Aegilops tauschii (Synthetic
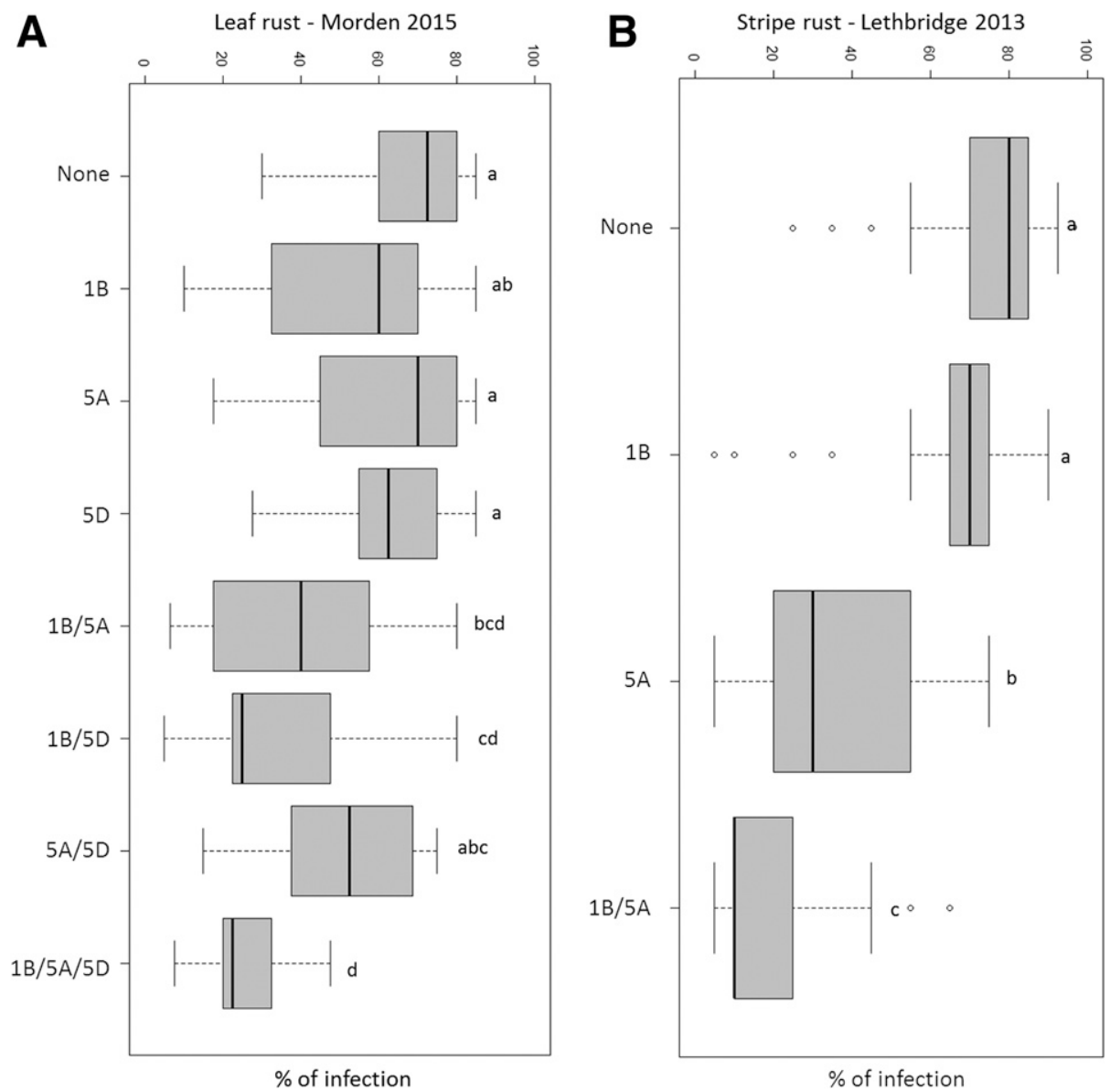

Fig. 4. The mean infection phenotypes of double haploid (DH) lines from the Toropi-6.4 $\times$ Thatcher population grouped by rust resistant quantitative trait loci (QTL). A, The mean leaf rust infection values for DH lines containing the following QTL combinations: none, QLr.crc-1BL (1B), QLr.crc-5AL (5A), QLr.crc-5DS (5D), QLr.crc-1BL plus QLr.crc-5AL (1B/5A), QLr.crc-1BL plus Lr.crc-5DS (1B/5D), QLr.crc-5AL plus QLr.crc-5DS (5A/5D), and all three QTL (1B/5A/5D). B, The mean stripe rust infection values for DH lines containing the following QTL combinations: none, QYr.crc-1BL (1B), QYr.crc-5AL (5A), and both QTL (1B/ 5A). The data shown are for the leaf rust field trial in Morden 2015 and the stripe rust field trial in Lethbridge 2013. Box plots show the median (black solid line), 25 and 75 percentile rust infection values (lower and upper limits of box). The white dots represent outlying data points. Lowercase letters that differ indicate QTL combinations that differ from each other. (All other leaf and stripe rust field trial analyses can be found in Supplementary Figures S4 and S5, respectively.) 
205)//Kauz) developed by CIMMYT (Mujeeb-Kazi et al. 2000). Yr34 was identified in an Australian wheat advanced breeding line and shown to be allelic, or very closely linked to $\mathrm{Yr} 48$ (Qureshi et al. 2018).

QYr.crc- $4 B L$ was only found in the Lethbridge 2018 trial. There are three $Y r$ gene described on chromosome 4BL, $\operatorname{Yr} 50, \operatorname{Yr} 62$, and Yr68 (McIntosh et al. 2008). Yr50 is an all-stage resistance gene derived from Thinopyrum intermedium (Liu et al. 2013). Yr62 and Yr68 are APR genes, and were identified in the wheat line PI 192252 (Lu et al. 2014) and Avocet S*5 (McIntosh et al. 2008), respectively. Yr62 is linked to the marker locus Xgwm251, which was also mapped in the Toropi- $6.4 \times$ Thatcher population, positioned $6.8 \mathrm{cM}$ from the peak marker defining QYr.crc-4BL. Yr68 is linked to marker IWA4640, the location of which on the International Wheat Genome Sequencing Consortium RefSeq v. 1.0 genome places it in the vicinity of $Q Y$ r.crc- $4 B L$

QYr.crc- $2 B S$, derived from Thatcher, was detected in the Kenya 2018 stripe rust field trial, where Thatcher exhibited resistance to stripe rust. The stripe rust resistance in Thatcher is probably due to the presence of $Y r 7$. Although virulence to $Y r 7$ is frequently found in Kenya (Mogens Hovmoller, personnel communication), the Kenyan $P$. striiformis $\mathrm{f}$. sp. tritici population carries alleles both virulent and avirulent to $Y r 7$. The location of the markers defining QYr.crc-2BS colocate with the KASP markers developed for $Y r 7$ (Marchal et al. 2018) as indicated by the International Wheat Genome Sequencing Consortium RefSeq v.1.0 genome.

The leaf and stripe rust resistance in Toropi represent a valuable resource for wheat rust resistance breeding. In addition to known leaf and stripe rust resistance loci such as $L r 46 / Y r 29$, we have identified potentially new sources of leaf rust resistance on the long arm of chromosome 5A, QLr.crc-5AL. The positive interactions between QLr.crc-1BL (Lr46/Yr29 locus) and QLr.crc-5AL and QLr.crc-5DS is of particular interest, the level of leaf rust resistance being greater than seen in lines containing QLr.crc-5AL and QLr.crc-5DS. The stripe rust resistance QTL QYr.crc-5AL is also worthy of further study. This QTL reduced stripe rust infection significantly in eight out of nine field trials, the level of stripe rust resistance varying considerably across locations and years.

\section{ACKNOWLEDGMENTS}

We thank P. Seto-Goh, M. Popovic, A. Brown, and E. Reimer, the former DH lab of AAFC-Winnipeg for excellent technical assistance. We also thank M. Chaves, Embrapa Clima Temperado, Pelotas, Brazil; S. Brammer, Embrapa Wheat, Passo Fundo, Brazil; J. Martinelli, Federal University of Rio Grande do Sul, Porto Alegre, Brazil; S. German, National Institute of Agricultural Research of Uruguay, Montevideo, Uruguay; and E. Lagudah, Commonwealth Scientific and Industrial Research Organisation, Canberra, Australia for supplying DNA of different seed lots of the wheat cultivar Toropi. We also thank S. Bhavani, CIMMYT, Nairobi, Kenya, for providing stripe rust data in Kenya 2012 and 2018. Contact B. D. McCallum to access seeds of Toropi-6, Toropi-6.3, and Toropi-6.4.

\section{LITERATURE CITED}

Allen, A. M., Barker, G. L. A., Berry, S. T., Coghill, J. A., Gwilliam, R., Kirby, S., Robinson, P., Brenchley, R. C., D'Amore, R., McKenzie, N., Waite, D., Hall, A., Bevan, M., Hall, N., and Edwards, K. J. 2011. Transcript-specific, single-nucleotide polymorphism discovery and linkage analysis in hexaploid bread wheat (Triticum aestivum L.). Plant Biotechnol. J. 9:1086-1099.

Barcellos, A. L., Roelfs, A. P., Moraes-Fernandes, M., and de Moraes-Fernandes, M. I. B. 2000. Inheritance of adult plant leaf rust resistance in the Brazilian wheat cultivar Toropi. Plant Dis. 84:90-93.

Broman, K. W., and Sen, S. 2009. A Guide to QTL Mapping with R/QTL. Springer, New York.

Caldwell, R. M. 1968. Breeding for general and/or specific plant disease resistance. Pages 263-272 in: Proc. 3rd Int. Wheat Genetics Symp. K. W. Finley and K. W. Shepherd, eds. Australian Academy of Science, Canberra, Australia.

Casassola, A., Brammer, S. P., Chaves, M. S., Martinelli, J. A., Stefanato, F., and Boyd, L. A. 2015. Changes in gene expression profiles as they relate to the adult plant leaf rust resistance in the wheat cv. Toropi. Physiol. Mol. Plant Pathol. 89:49-54.

Chao, S., and Somers, D. 2012. Wheat and barley DNA extraction in 96-well plates. University of California. https://maswheat.ucdavis.edu/

Da-Silva, P. R., Brammer, S. P., Guerra, D., Milach, S. C. K., Barcellos, A. L., and Baggio, M. I. 2012. Monosomic and molecular mapping of adult plant leaf rust resistance genes in the Brazilian wheat cultivar Toropi. Genet. Mol. Res. 11:2823-2834.

Dyck, P. L. 1987. The association of a gene for leaf rust resistance with the chromosome 7D suppressor of stem rust resistance in common wheat. Genome 29:467-469.

Gupta, S. K., Charpe, A., Koul, S., Haque, Q. M. R., and Prabhu, K. V. 2006. Development and validation of SCAR markers co-segregating with an Agropyron elongatum derived leaf rust resistance gene $\mathrm{Lr} 24$ in wheat. Euphytica 150:233-240.

Hare, R. A., and McIntosh, R. A. 1979. Genetic and cytogenetic studies of durable adult-plant resistances in 'Hope' and related cultivars to wheat rusts. Z. Pflanzenzucht. 83:350-367.

Herrera-Foessel, S. A., Lagudah, E. S., Huerta-Espino, J., Hayden, M., Bariana, H. S., Singh, D., and Singh, R. P. 2011. New slow rusting leaf rust and stripe rust resistance genes $\operatorname{Lr} 67$ and $Y r 46$ in wheat are pleiotropic or closely linked. Theor. Appl. Genet. 122:239-249.

Herrera-Foessel, S. A., Singh, R. P., Huerta-Espino, J., Rosewarne, G. M., Periyannan, S. K., Viccars, L., Calvo-Salazar, V., Lan, C. X., and Lagudah, E. S. 2012. Lr68: A new gene conferring slow rusting resistance to leaf rust in wheat. Theor. Appl. Genet. 124:1475-1486.

Hiebert, C. W., Thomas, J. B., McCallum, B. D., Humphreys, D. G., DePauw, R. M., Hayden, M. J., Mago, R., Schnippenkoetter, W., and Spielmeyer, W. 2010. An introgression on wheat chromosome 4DL in RL6077 (Thatcher*6/ PI 250413) confers adult plant resistance to stripe rust and leaf rust (Lr67). Theor. Appl. Genet. 121:1083-1091.

Kassa, M. T., You, F. M., Fetch, T. G., Fobert, P., Sharpe, A., Pozniak, C. J., Menzies, J. G., Jordan, M. C., Humphreys, G., Zhu, T., Luo, M. C., McCartney, C. A., and Hiebert, C. W. 2016. Genetic mapping of SrCad and SNP marker development for marker-assisted selection of Ug99 stem rust resistance in wheat. Theor. Appl. Genet. 129:1373-1382.

Knott, D. R. 1989. The Wheat Rusts-Breeding for Resistance. Springer, Berlin, Heidelberg.

Kolmer, J. A. 2015. A QTL on chromosome 5BL in wheat enhances leaf rust resistance of Lr46. Mol. Breed. 35:74.

Kolmer, J. A., Bernardo, A., Bai, G., Hayden, M. J., and Chao, S. 2018. Adult plant leaf rust resistance derived from Toropi wheat is conditioned by $\mathrm{Lr} 78$ and three minor QTL. Phytopathology 108:246-253.

Kosambi, D. D. 1943. The estimation of map distances from recombination values. Ann. Eugen. 12:172-175.

Krattinger, S. G., Lagudah, E. S., Spielmeyer, W., Singh, R. P., Huerta-Espino, J., McFadden, H., Bossolini, E., Selter, L. L., and Keller, B. 2009. A putative $\mathrm{ABC}$ transporter confers durable resistance to multiple fungal pathogens in wheat. Science 323:1360-1363.

Lan, C., Zhang, Y., Herrera-Foessel, S. A., Basnet, B. R., Huerta-Espino, J., Lagudah, E. S., and Singh, R. P. 2015. Identification and characterization of pleiotropic and co-located resistance loci to leaf rust and stripe rust in bread wheat cultivar Sujata. Theor. Appl. Genet. 128:549-561.

Li, C., Wang, Z., Li, C., Bowden, R., Bai, G., Su, Z., and Carver, B. F. 2017. Mapping of quantitative trait loci for leaf rust resistance in the wheat population Ning7840 $\times$ Clark. Plant Dis. 101:1974-1979.

Lillemo, M., Asalf, B., Singh, R. P., Huerta-Espino, J., Chen, X. M., He, Z. H., and Bjørnstad, A. 2008. The adult plant rust resistance loci $\operatorname{Lr} 34 / Y r 18$ and $L r 46 / Y r 29$ are important determinants of partial resistance to powdery mildew in bread wheat line Saar. Theor. Appl. Genet. 116:1155-1166.

Liu, J., Chang, Z., Zhang, X., Yang, Z., Li, X., Jia, J., Zhan, H., Guo, H., and Wang, J. 2013. Putative Thinopyrum intermedium-derived stripe rust resistance gene Yr50 maps on wheat chromosome arm 4BL. Theor. Appl. Genet. 126:265-274

Lorieux, M. 2012. MapDisto: Fast and efficient computation of genetic linkage maps. Mol. Breed. 30:1231-1235.

Lowe, I., Jankuloski, L., Chao, S., Chen, X., See, D., and Dubcovsky, J. 2011. Mapping and validation of QTL which confer partial resistance to broadly virulent post-2000 North American races of stripe rust in hexaploid wheat. Theor. Appl. Genet. 123:143-157.

Lu, Y., Wang, M., Chen, X., See, D., Chao, S., and Jing, J. 2014. Mapping of Yr62 and a small-effect QTL for high-temperature adult-plant resistance to stripe rust in spring wheat PI 192252. Theor. Appl. Genet. 127:1449-1459.

Marchal, C., Zhang, J., Zhang, P., Fenwick, P., Steuernagel, B., Adamski, N. M., Boyd, L., McIntosh, R., Wulff, B. B. H., Berry, S., Lagudah, E., and Uauy, C. 2018. BED-domain-containing immune receptors confer diverse resistance spectra to yellow rust. Nat. Plants 4:662-668.

McIntosh, R. A. 1992. Close genetic linkage of genes conferring adult-plant resistance to leaf rust and stripe rust in wheat. Plant Pathol. 41:523-527. 
McIntosh, R. A., Wellings, C., and Park, R. 1995. Wheat Rusts: An Atlas of Resistance Genes. Kluwer Academic Publishers, Dordrecht.

McIntosh, R. A., Yamazaki, Y., Dubcovsky, J., Rogers, J., Morris, C., Somers, D. J., Appels, R., and Devos, K. M. 2008. Catalogue of gene symbols for wheat. National BioResource Project, Komugi-Wheat Genetic Resources. https://shigen.nig.ac.jp/wheat/komugi/genes/download.jsp

Mendiburu, F. 2017. Package "agricolae" statistical procedures for Agricultural Research. https://cran.r-project.org/web/packages/agricolae/index.html

Meng, L., Li, H., Zhang, L., and Wang, J. 2015. QTL IciMapping: Integrated software for genetic linkage map construction and quantitative trait locus mapping in biparental populations. Crop J. 3:269-283.

Moore, J. W., Herrera-Foessel, S., Lan, C., Schnippenkoetter, W., Ayliffe, M., Huerta-Espino, J., Lillemo, M., Viccars, L., Milne, R., Periyannan, S., Kong, X., Spielmeyer, W., Talbot, M., Bariana, H., Patrick, J. W., Dodds, P., Singh, R., and Lagudah, E. 2015. A recently evolved hexose transporter variant confers resistance to multiple pathogens in wheat. Nat. Genet. 47: 1494-1498.

Mujeeb-Kazi, A., Gilchrist, L. I., Villareal, R. L., and Delgado, R. 2000. Registration of ten wheat germplasm resistant to leaf blotch. Crop Sci. 40: 590-591.

Peterson, R. F., Campbell, A. B., and Hannah, A. E. 1948. A diagrammatic scale for estimating rust intensity on leaves and stems of cereals. Can. J. Res. C 26:496-500.

Pinto da Silva, G. B., Zanella, C. M., Martinelli, J. A., Chaves, M. S., Hiebert, C. W., McCallum, B. D., and Boyd, L. A. 2018. Quantitative trait loci conferring leaf rust resistance in hexaploid wheat. Phytopathology 108: 1344-1354.

Pretorius, Z. A., Ayliffe, M., Bowden, R. L., Boyd, L. A., DePauw, R. M., Jin, Y., Knox, R. E., McIntosh, R. A., Park, R. F., Prins, R., and Lagudah, E. 2017. Advances in control of wheat rusts. In: Achieving Sustainable Cultivars of Wheat Volumes 1 and 2 Ed. Langridge P. Burleigh Dodds Publishers, Cambridge, UK.

Qureshi, N., Bariana, H. S., Zhang, P., McIntosh, R., Bansal, U. K., Wong, D., Hayden, M. J., Dubcovsky, J., and Shankar, M. 2018. Genetic relationship of stripe rust resistance genes $\mathrm{Yr} 34$ and $\mathrm{Yr} 48$ in wheat and identification of linked KASP markers. Plant Dis. 102:413-420.

R Development Core Team. 2018. R: A language and environment for statistical computing. R Foundation for Statistical Computing, Vienna, Austria. http://www.R-project.org

Revelle, W. 2018. psych: Procedures for personality and psychological research, Version $=1.8 .12$. Northwestern University, Evanston, IL. https:// cran.r-project.org/web/packages/psych/index.html

Roelfs, A. P., Singh, R. P., and Saari, E. E. 1992. Rust Diseases of Wheat: Concepts and Methods of Disease Management. CIMMYT, Mexico.

Rosa, S. B., McCallum, B., Brûlé-Babel, A., Hiebert, C., Shorter, S., Randhawa, H. S., and Barcellos, A. L. 2016. Inheritance of leaf rust and stripe rust resistance in the Brazilian wheat cultivar Toropi. Plant Dis. 100:1132-1137.

Rosewarne, G. M., Herrera-Foessel, S. A., Singh, R. P., Huerta-Espino, J., Lan, C. X., and He, Z. H. 2013. Quantitative trait loci of stripe rust resistance in wheat. Theor. Appl. Genet. 126:2427-2449.

Rosewarne, G. M., Singh, R. P., Huerta-Espino, J., Herrera-Foessel, S. A., Forrest, K. L., Hayden, M. J., and Rebetzke, G. J. 2012. Analysis of leaf and stripe rust severities reveals pathotype changes and multiple minor QTL associated with resistance in an Avocet $\times$ Pastor wheat population. Theor. Appl. Genet. 124:1283-1294.

Rosewarne, G. M., Singh, R. P., Huerta-Espino, J., William, H. M., Bouchet, S., Cloutier, S., McFadden, H., and Lagudah, E. S. 2006. Leaf tip necrosis, molecular markers and $\beta 1$-proteasome subunits associated with the slow rusting resistance genes Lr46/Yr29. Theor. Appl. Genet. 112:500-508.

Singh, R. P. 1992. Genetic association of leaf rust resistance gene Lr34 with adult plant resistance to stripe rust in bread wheat. Phytopathology 82: 835-838.

Singh, R. P., Herrera-Foessel, S. A., Huerta-Espino, J., Lan, C. X., Basnet, B. R., Bhavani, S., and Lagudah, E. S. 2013. Pleiotropic gene Lr46/Yr29/ Pm39/Ltn2 confers slow rusting, adult plant resistance to wheat stem rust fungus. Page 17.1 in: Proceedings Borlaug global rust initiative, 2013 Technical Workshop, New Delhi.

Singh, R. P., Huerta-Espino, J., and Rajaram, S. 2000. Achieving nearimmunity to leaf and stripe rusts in wheat by combining slow rusting resistance genes. Acta Phytopathol. Hun. 35:133-139.

Singh, R. P., Huerta-Espino, J., and William, H. M. 2005. Genetics and breeding for durable resistance to leaf and stripe rusts in wheat. Turk. J. Agric. 29:121-127.

Singh, R. P., Payne, T. S., and Rajaram, S. 1991. Characterization of variability and relationship among components of partial resistance to leaf rust in CIMMYT bread wheats. Theor. Appl. Genet. 82:674-680.

Singh, R. P., and Rajaram, S. 1992. Genetics of adult-plant resistance to leaf rust in 'Frontana' and three CIMMYT wheats. Genome 35:24-31.

Somers, D. J., Isaac, P., and Edwards, K. 2004. A high-density microsatellite consensus map for bread wheat (Triticum aestivum L.). Theor. Appl. Genet. 109:1105-1114.

Spielmeyer, W., McIntosh, R. A., Kolmer, J., and Lagudah, E. S. 2005. Powdery mildew resistance and $\mathrm{Lr} 34 / \mathrm{Yr} 18$ genes for durable resistance to leaf and stripe rust cosegregate at a locus on the short arm of chromosome 7D of wheat. Theor. Appl. Genet. 111:731-735.

Tang, Y., Horikoshi, M., and Li, W. 2016. ggfortify: Unified interface to visualize statistical result of popular R Packages. R Journal 8:474-489.

Thomas, J., Nilmalgoda, S., Hiebert, C., McCallum, B., Humphreys, G., and DePauw, R. 2010. Genetic markers and leaf rust resistance of the wheat gene Lr32. Crop Sci. 50:2310-2317.

Voorrips, R. E. 2002. MapChart: Software for the graphical presentation of linkage maps and QTLs. J. Hered. 93:77-78.

Wang, M., and Chen, X. 2017. Stripe rust resistance. In: Stripe Rust. X.Chen and Z. Kang, eds. Springer, Dordrecht, The Netherlands.

Wang, S., Wong, D., Forrest, K., et al. 2014. Characterization of polyploid wheat genomic diversity using a high-density 90,000 single nucleotide polymorphism array. Plant Biotechnol. J. 12:787-796.

Wei, T., and Simko, V. 2017. R package "corrplot": Visualization of a Correlation Matrix (Version 0.84). https://github.com/taiyun/corrplot

Wesp-Guterres, C., Martinelli, J. A., Graichen, F. A. S., and Chaves, M. S. 2013. Histopathology of durable adult plant resistance to leaf rust in the Brazilian wheat variety Toropi. Eur. J. Plant Pathol. 137:181-196.

Wickham, H. 2016. ggplot2: Elegant Graphics for Data Analysis. SpringerVerlag, New York.

Zoldan, S. M., and Barcellos, A. L. 2002. Postulação de genes (Lr) de resistência à ferrugem da folha em cultivares brasileiras de trigo. Fitopatol. Bras. 27:508-516. 